\title{
Robust Control for a Noncolocated Spring-Mass System
}

\author{
Richard D. Brastz"and Manfred Morari ${ }^{\dagger}$ \\ Chemical Engineering 210-41, California Institute of Technology, Pasadena, CA 91125
}

\section{Abstract}

Robust control laws are preacated for an undamped pair of coupled manes with a noncolocated ensor and setuator. This simple problem captures many of the features of more complex aircraft and space strueture vibration control problems. The control probtem is formulated in the structured singular value framework, which addremes the stability robustnes to parameter variations direetly. Controllen are desigmed by D-K iteration (commonly called (1-synthesis), and the resulting high-order controllers are reduced us ing Hankel model reduction. Deaiga apecifications wech as settling time, setuator constraints, imenestivity to meesurement noive, and perameter uncertainty are ectrieved by the resulting controllers. Deaign Problems \#1 and \#2 were considered in [2]. Design Problem \#4 in [11] will be considered in this paper.

Introduction

Numerous researchers (a listed in [11]) have applied a variety of robust control design methodologies to the benchmark problem. Brasts and Morari [2] desimed robust controlkers for Desion Problems \#1 and \#2 using the "DK iteration" method propoed by Doyle [6]. Though the design specifications cannot be described directly in the arractured singular value framewort, control, performance, dis turbance, and measurement weights were choeen to meet the design apecification.

The paper organized as follows. Firat the benchmark problem is briefly deacribed. Then the etructured singular value framework is reviewed. Design Problem *4 is then put into this framework. Due to lack of space, the state-apace matrices for the controllex, the gain and phane margins, the performance/Atability robustoes plots, and time simulations are not given here but will be presented at the 1992 ACC Conference [3].

\section{Beschmark Problem}

Consider the trro-man/spring system in [11], which is a generic model of an uncertsin dyaamical system with noncolocated sensor and actuator. The system is represented in atate-space form as

$$
\begin{aligned}
& i=\left[\begin{array}{l}
\dot{x}_{1} \\
\dot{x}_{2} \\
\dot{x}_{3} \\
\dot{x}_{4}
\end{array}\right]=\left[\begin{array}{cccc}
0 & 0 & 1 & 0 \\
0 & 0 & 0 & 1 \\
-k / m_{1} & k / m_{1} & 0 & 0 \\
k / m_{2} & -k / m_{2} & 0 & 0
\end{array}\right]\left[\begin{array}{l}
x_{1} \\
x_{2} \\
x_{3} \\
x_{4}
\end{array}\right]+\left[\begin{array}{c}
0 \\
0 \\
1 / m_{1} \\
0
\end{array}\right] u+\left[\begin{array}{c}
0 \\
0 \\
0 \\
1 / m_{2}
\end{array}\right] w \\
& y=x_{2}+v \\
& z=z_{2}
\end{aligned}
$$

where $x_{1}$ and $x_{2}$ are the positions of body 1 and body $2, x_{3}$ and $x_{1}$ are the velocities of body 1 and body $2, x$ is the control input acting on body $1, y$ is the sensor mewarement, $w$ is the disturbance acting on body $2, v$ is seneor noise, and $z$ in the output to be controlled. $k$ is the spring constant, $m_{1}$ is the mass of body 1 , and $m_{2}$ is the mass of body 2.

The coupled apring-mass aystem is acoumed to have negligible damping. The spring constant and manses are asumed to be uncertwin. The actuator is located on body 1 while the sensor is located on body 2, i.e. the seneor and actuator are soncolocated. This makes the system much harder to control than in the colocated case.

Design Problem \#4 is described betor. Specifeations $(i-i v)$ are from [11]. Specifications $(v-v i)$ from [2] are additional practical constraints. We choose the measurement noise to be approximately the same as that for the laboratory lexible structure in [1]. Our actuator band ridth limitation is more reatrictive than that for the voice coil actuators in [1].

Deaign *4. Design a feedback/feedforward controller for a unitstep output command tracking problem for the controlled output, $z$, with the following properties:

(i) The control input $u(t)$ is limited to $|u| \leq 1$.

(ii) Performance requirement: settling time and overshoot are both to be minimised.

(iii) Stability requirement: performance robustness and stability robustness with respect to the three uncertain parameters $m_{1}, m_{2}$, and $k$ (with the nominal values $m_{1}=m_{2}=k=1$ ) are both to marimised.

(iv) If there are conflicts between (2) and (3), then performance versus robustness trade-ofis must be considered.

(v) The control system can tolerate Gaussian white noise with variance of $9 * 10^{-6}$

- eupported by the Fannie and John Herts Formdation

1To whom correcpondence should be addresed: phone (818)356-4186, fax (818)568-8743, e-mil mm Pimc.caltoch.edu (vi) Because of finite actuator response time, the bandwidth for the feedback controller mut be $\leq 50 \mathrm{rad} / \mathrm{s}$.

\section{Structured Singular Value Framework}

The goal of any controllex decigon is that the overall system is stable and antiffes some minimum performance requirements. These requirements should be satiafied at least when the controller is applied to the nominal plant, that is, we require nominal stability and nominal performance.

In practice the real plant $G_{p}$ is not equal to the model $G$. The term "robust" is used to indicate that some property bolds for a set II of pousible plants $G$, as defined by the uncertainty description. In particular, by robust stability we mean that the cloeed loop system is atable for all $G, \in$ II. By robuat performance we mean that the performance requirements are ativfied for all $G, \in$ II. Performance is commonly defined in robust control theory uing the $H_{\infty}$-norm of some trander function $\Sigma(G)$ of interest.

Definition 1 The clased loop oystem exhibits nominal performance if

$$
\|\Sigma\|_{\infty} \equiv \operatorname{mp} \sigma(\Sigma) \leq 1 \text {. }
$$

Definition 2 The closed loop sostem exhibits robest performence if

$$
\left\|\Sigma_{p}\right\|_{\infty} \equiv \sup \sigma\left(\Sigma_{p}\right) \leq 1, \quad \forall G, \in \text { I. }
$$

For example, for rejection of diaturbances at the plant output, $\Sigma$ would be the weighted sensitivity

$$
\begin{array}{ll}
\Sigma=W_{1} S W_{2}, & S=(I+G K)^{-1} \\
\Sigma_{2}=W_{1} S_{p} W_{2}, & S_{\mathrm{p}}=\left(I+G_{p} K\right)^{-1} .
\end{array}
$$

In this case, the input weight $W_{2}$ is usually choeen equal to the disturbance model. The output meight $W_{1}$ is unod to specify the frequency range over which the ensitivity function should be small and to weight each output according to its importance. $K$ is the transer function of the controller.

Doyle [ 4 ] derived the struetured ringeler value, $\mu$, to teat for robuat performance. To use $\mu$ we mut model the uncertsinty (the set II of poesible plants $G_{p}$ ) as norm bounded perturbations $\left(\Delta_{i}\right)$ on the nominal system. Through weights each perturbation is normalized to be of size one:

$$
\bar{\sigma}\left(\Delta_{i}\right) \leq 1, \quad \forall \omega .
$$

The perturbations, whieh mat occur at different locations in the system, are collected in the diagonal matrix $\Delta_{U}=\operatorname{diag}\left[\Delta_{1}, \cdots, \Delta_{n}\right]$ (the $U$ denotes uncertainty) and the system is arranged to mateh the block diagrams in Fig. 1. The interconnection matrix $M$ in Fig. 1 is determined by the nominal model $(G)$, the sice and nature of the uncertainty, the performance specifications, and the controller $(K)$. The definition of $\mu$ is:

Definition 3 Let $M$ be a square complex matrix and the set $\Delta=$ $\left\{\operatorname{diag}\left[\Delta_{1}, \cdots, \Delta_{n}\right]\right\}$. Then $\mu_{\Delta}(M)$ is defined such thet $\mu_{\Delta}^{-1}(M)$ is equal to the smallest $\bar{\sigma}(\Delta)$ for $\Delta$ making $(I+\Delta M)$ singular, i.e.

$$
\mu_{\Delta}^{-1}(M)=\min _{\Delta \in \Delta}\{\sigma(\Delta): \operatorname{det}(I+\Delta M)=0\} .
$$

For Fig. 1, robust stability and robust performance can be tested by Theorem 1 The elosed loop system exhibits robust stebility if and only if the closed loop syotem is nominally steble and

$$
\sup \mu_{\Delta_{v}}\left(M_{11}\right)<1
$$

Theorem 2 The closed loop system exhitits robust performexce if end oanty if the closed loop system is aominelly stable and

$$
\sup _{\mathbf{\Delta}} \mu_{\Delta}(\boldsymbol{M})<1 \text {. }
$$

$\mu_{\Delta}(M)$ depende on both the elements of the matrix $M$ and the structure of the perturbation matrix $\Delta=\operatorname{diag}\left[\Delta_{U}, \Delta_{P}\right] . \Delta_{P}$ is often chooen to be a full square matrix with dimension equal to the number of outputs (the subecript $P$ denotes performance). Note that the issue of robust stability is simply a special case of robust performance. Also note that robust performance implies robust stability, i.e. $\sup \mu_{\Delta}(M) \geq \sup \mu_{\Delta_{U}}\left(M_{11}\right)$.

It is a key idea that $\mu$ is a general analysis tool for determining robust performance. Any system with uncertainty adequately modeled as in (7) can be put into $M-\Delta$ form, and robust performance can be tested using (10). Standard programs cal culate $M$ and $\Delta[7]$, given the transfer fuactions describing the system components and the location of the uncertainty blocks $\Delta_{i}$. 
Upper Bound for $\mu$ with Complex $\Delta$ Because calculating $\mu$ exactly is fairly difficult, its weltknown upper bound is used instead. Define

$\mathbf{D}=\left\{\operatorname{diag}\left[d_{i} I_{i}\right]: \operatorname{dim}\left(I_{i}\right)=\operatorname{dim}\left(\Delta_{i}\right), d_{i}\right.$ pocitive real scalar $\}$,

then [4]

$$
H_{\Delta}(M) \leq \inf _{D \in D} \bar{\sigma}\left(D M D^{-1}\right) .
$$

The upper bound is almost always within a percent or so of $\mu$ for real problems [10], $s$ for engineering purposes $\mu$ never has to be calculated exactly.

Controller Synthesis $M$ is s function of the controller $K$. The $H_{\infty}$ optimal control problem is to find a stabilising $K$ which minimires sup $\bar{\sigma}(M(K))$. The etate-epece approsch for oolviag the $H_{\infty}$ control

roblem in dencribed in [9]

The D-K iteration method (often called p-syntheais) is an ed hoc method which attempts to minimize the tight upper bound of $\mu$ in (12), i.e. it attempts to solve

$$
\min _{K} \inf _{D \in D} \operatorname{aup} \sigma\left(D M(K) D^{-1}\right) \text {. }
$$

The approach in D-K iteration is to alternatively minimize eup. $\delta\left(D M(K) D^{-1}\right)$ for either $K$ or $D$ while holding the other constant. For fixed $D$, the controller synthesis is solved via $H_{\infty}$-optimization. For fixed $K$, the quantity is minimised as a conver optimiration. The resulting $D$ as a function of frequency is fitted with an invertible atable minimumphase tranerer function and wrapped back into the nominal interconnection etructure. This increases the number of atates of the scaled $G$, which leads the next $H_{\infty}$-oynthesis step to give a higher order controller. The iterations stop after $\sup _{\omega} \approx\left(D M(K) D^{-1}\right)$ is no longer diminished. The resulting high-order controller is reduced using Hankel model reduction [8]. Though this method is not guaranteed to converge to a global minimum, it has been ueed extensively to design robut controllers and seems to work well [5].

Application of $\mu$ to Dexign Problem \#4

The spring constant and the two mases are asumed to be uncertain and are given by

$k=k_{0}+w_{k} \delta_{k}, \quad m_{1}=m_{10}+w_{1} \delta_{1}, \quad m_{2}=m_{20}+w_{2} \delta_{2}$, where $k_{0}, m_{10}$, and $m_{20}$ are the nominal values and the weights $w_{k}$, $w_{1}$, and $w_{2}$ are used to normalize the uncertainties $\delta_{i}$ wo that $\left|\delta_{i}\right| \leq 1$. Simultaneous perturbations in the $\delta_{i}$ are allowed, os long $\approx\left|\delta_{i}\right| \leq 1$ for each uncertainty $i$.

The objective of Design Problem \#4 is for the controlled variable $z$ to follow the desired trajectory $r$, i.e. we want $\|z-r\|_{\infty}$ small. Weighted veraions of the noise, disturbance, control input, and performance variable are given by

$r=w_{r} r^{\prime}, \quad w=w_{w} w^{\prime}, \quad u^{\prime}=w_{u} u, \quad(z-r)^{\prime}=w_{z r}(z-r), \quad$ (15) where in general the input weights $w_{r}$ and $w_{w}$ weigh the frequencies of interest and determine the relative importance of the noise and disturbance. $w_{3 r}$ is the performance weight and $w_{n}$ is used to limit the magnitude of the control input. It is suspected that the high frequeney roll-of required by specification (vi) will automatically force specification $(v)$ to be satisfied, $s 0(v)$ is not directly accounted for in the controller synthesis. Specification (v) will be checked in the time simulations.

$k, m_{1}$ and $m_{2}$ from (14) and $r, w, u$, and $z$ from (15) are subetituted into the state-space equations (1-3) and written in block diagram form in Fig. 2 . The block diagram has $x, f, w^{\prime}, u$ as inputs and $\dot{x}, u^{\prime},(z-r)^{\prime}, r$, and $y$ as outputs.

By inspection, the block diagram in Fig. 2 is rearranged to form the block diagram in Fig. 3, where

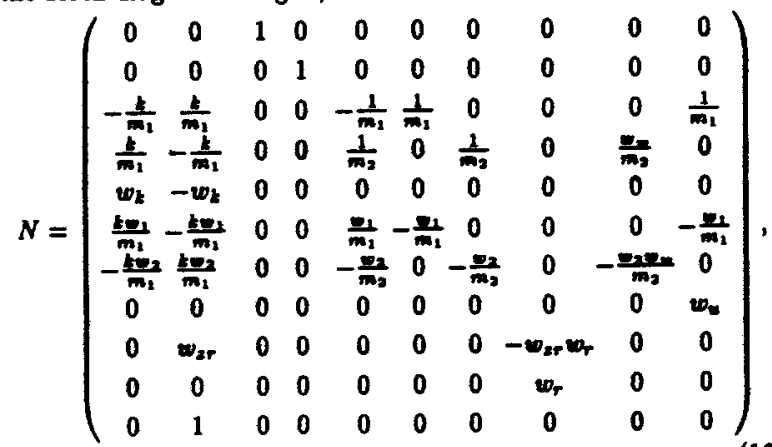

and the normalized performance variable $\hat{e}$, the normalized disturbance $\hat{d}$, and the uncertainty block $\Delta_{U}$ are given by

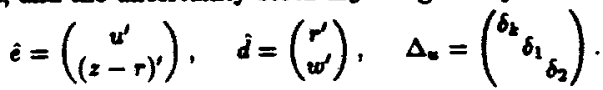

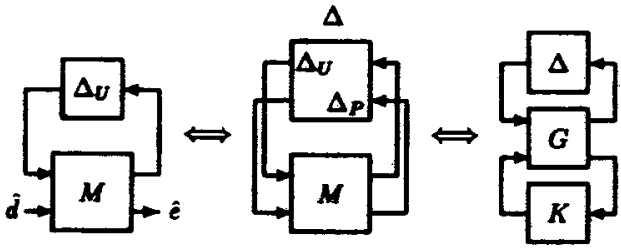

Figure 1: General Interconnection Structures

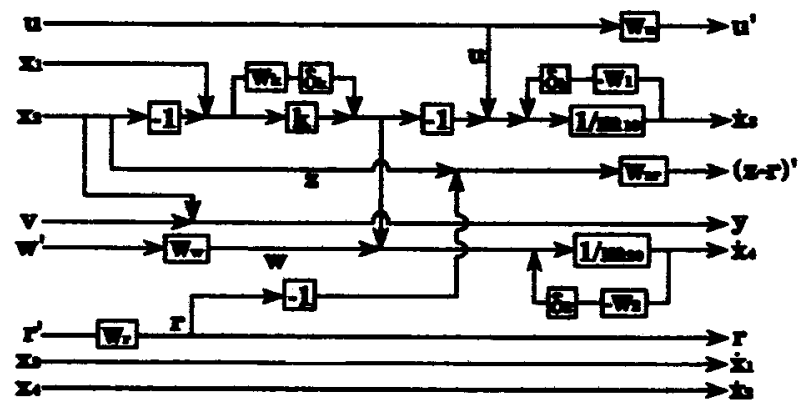

Figure 2: Block Diagram for Coupled Maso-Spring System

$\Delta_{P}$ is the performance $\Delta$-block which relates the outputs to inputs, $K$ is the controller transfer function, and $I_{4}$ is the $4 \times 4$ identity matrix. Closing the integrator loop in Fis. 3 gives the system interconnection structures in Fig. 1. Note that $K$ has two inputo the reference trajectory $r$ and the measured variable $y . K=\left[K_{F F} K_{F_{B}}\right]$, where $K_{F F}$ is the feedforward and $K_{F B}$ is the feedback controller.

We will wee the D-K iteration method by allowing the uncertainties in $k, m_{1}$, and $m_{2}$ to be complex. The D-K iteration method described in Section 3 approximately maximizes the performance for the worst-case plant described by the nominal plant plus the complex uncertainties. As such, the method will give a controller whose performance is insensitive to the complex uncertainties. Thus the performance of the controller will also be insensitive to the corresponding real uncertainties.

Due to lack of space, the choice of weights, the state-space mar trices for the controller, the gain and phase margins, the performance/stability robustness plots, and time simulations are not given here but will be presented at the 1992 ACC Conference [3].

References

[1]G. J. Balas. PhD thesis, Caltech, Pasadena, 1990.

[2]R. D. Brasts and M. Morari. AIA JGCD, 1992. in publication.

[3]R. D. Braste and M. Morari. In ACC Proc., 1992.

[4]J. C. Doyke. IEE Proc. Pert D, pages 242-250, 1982.

[5]J. C. Doyle. ONR/Honegwell Leeture Notes. Minneapolis, 1984.

[6]J. C. Doyle. In CDC Proc., pages 260-265, 1985.

[7] G. J. Balas et al. In ACC Proc., pages 996-1001, 1991.

[8]K. Glover. IJC, pages 1115-1193, 1984.

[9]K. Glover and J. C. Doyle. A state epace approach to $H_{\infty}$ optimal control. In Lectere Notes in Control and Information Sciences, volume 135. Springer-Veriag, 1989.

[10] A. K. Packard. PhD thecis, UC Berteley, 1988.

[11]B. Wie and D. S. Bernotein. Benchmart problems for robuet control design. In ACC Proc., 1992.

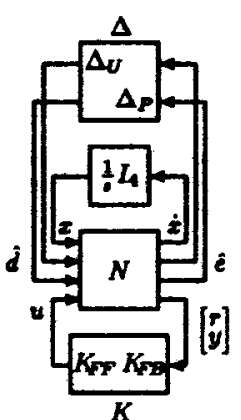

Figure 3: Simplified Block Diagram for Coupled Mas-Spring Syotem 